\title{
Effects of septal cholinergic lesion on rat exploratory behavior in an open-field
}

M.R. Lamprea ${ }^{1}$, F.P. Cardenas ${ }^{1}$, R. Silveira ${ }^{1}$, T.J. Walsh ${ }^{2+}$ and S. Morato ${ }^{1}$
${ }^{1}$ Faculdade de Filosofia, Ciências e Letras de Ribeirão Preto, Universidade de São Paulo, Ribeirão Preto, SP, Brasil

${ }^{2}$ Department of Psychology, Rutgers University, Piscataway, NJ, USA

\author{
Correspondence \\ S. Morato \\ Faculdade de Filosofia, Ciências \\ e Letras de Ribeirão Preto, USP \\ Av. Bandeirantes, 3900 \\ 14040-901 Ribeirão Preto, SP \\ Brasil \\ Fax: +55-16-633-5668 \\ E-mail: smorato@ffclrp.usp.br \\ Research partially supported by \\ grants from CNPq (No. 523094/95-7), \\ FAPESP (No. 98/11187-2) and NSF \\ IBN (No. 9514557). M.R. Lamprea \\ and F.P. Cardenas were the recipients \\ of fellowships from CAPES and \\ FAPESP, respectively. \\ ${ }^{\dagger}$ In memoriam.
}

Received March 27, 2002 Accepted December 12, 2002

\begin{abstract}
The medial septum participates in the modulation of exploratory behavior triggered by novelty. Also, selective lesions of the cholinergic component of the septohippocampal system alter the habituation of rats to an elevated plus-maze without modifying anxiety indices. We investigated the effects of the intraseptal injection of the cholinergic immunotoxin $192 \mathrm{IgG}$-saporin (SAP) on the behavior of rats in an open-field. Thirty-nine male Wistar rats (weight: 194-230 g) were divided into three groups, non-injected controls and rats injected with either saline $(0.5 \mu \mathrm{l})$ or SAP $(237.5 \mathrm{ng} / 0.5 \mu \mathrm{l})$. Twelve days after surgery, the animals were placed in a square open-field $(120 \mathrm{~cm})$ and allowed to freely explore for $5 \mathrm{~min}$. After the test, the rats were killed by decapitation and the septum, hippocampus and frontal cortex were removed and assayed for acetylcholinesterase activity. SAP increased acetylcholinesterase activity in the septum, hippocampus and frontal cortex and decreased the total distance run $(9.15 \pm 1.51 \mathrm{~m})$ in comparison to controls $(13.49 \pm 0.91 \mathrm{~m})$. The time spent in the center and at the periphery was not altered by SAP but the distance run was reduced during the first and second minutes $(2.43 \pm 0.36$ and $1.75 \pm 0.34 \mathrm{~m})$ compared to controls $(4.18 \pm 0.26$ and $3.14 \pm 0.25 \mathrm{~m})$. SAP-treated rats showed decreased but persistent exploration throughout the session. These results suggest that septohippocampal cholinergic mechanisms contribute to at least two critical processes, one related to the motivation to explore new environments and the other to the acquisition and storage of spatial information (i.e., spatial memory).
\end{abstract}

Key words

- Exploratory behavior

- Open-field

- Cholinergic immunotoxin

- Medial septum

- Saporin

- Acetylcholinesterase
Cholinergic neurons in the medial septum receive afferents from a variety of brainstem and midbrain areas that participate in arousal, motivation, and vegetative function (1). These neurons seem to integrate subcortical information and the projections of these neurons to the hippocampus through the septohippocampal cholinergic pathway modulate the hippocampal responsiveness to its primary cortical input, i.e., the entorhinal cortex (2). This projection has been implicated in a variety of behavioral processes including learning, anxiety, motivation, exploratory and ingestive behaviors (3). Lesions of this pathway as well as systemic or intrahippocampal administration of muscarinic antagonists produce deficits in a variety of cognitive tasks $(3,4)$. Kohler and Srebro (5) showed that electrolytic lesions of the medial septum abolished the exploration of 
an open-field accessible from the home cage and reduced locomotor activity. Similarly, medial septal lesions have been reported to decrease the exploration of both neutral and novel objects as well as rearing and locomotor activity levels in the open-field (6). Additionally, it has been demonstrated that lesions of the medial septum decrease the total activity in an open-field, especially in the central area, as well as exploratory behavior (7). It is interesting to note that other reports by these investigators $(8,9)$ as well as by others (10) consider increases in the activity in the central area of the open-field to be an index of decreased anxiety. Recently, we reported that intraseptal microinjections of the cholinergic immunotoxin 192 IgGsaporin (SAP) decreased exploratory behavior in an elevated plus-maze without affecting behaviors related to anxiety. In addition, a minute-by-minute analysis showed a deficit in habituation of exploratory behaviors (11).

The present study also utilized injections of the specific cholinergic immunotoxin 192 IgG-SAP. Immunotoxins are conjugates of a monoclonal antibody targeting a specific antigen combined with a ribosome-inactivating protein (12). Cholinergic neurons in the medial septum contain p75 neurotrophin receptors that contribute to the cellular effects of nerve growth factor and other trophic factors. SAP combines the 192 IgG monoclonal antibody to the p75 low affinity neurotrophin receptor, with the potent ribosomeinactivating protein SAP, derived from the plant Saponaria officinalis (13). Since all cholinergic cells in the medial septum express $\mathrm{p} 75$ receptors, site-specific injection of SAP into this area selectively destroys this cell population. Intraseptal injection of SAP produces dose-related a) loss of cholinergic neurons in the medial septum, b) regionally specific decreases in cholinergic indices in the targets of the septum (hippocampus, cingulate and entorhinal cortices), and c) delaydependent working memory impairments (14).
After demonstrating the role of the septohippocampal cholinergic pathway in habituation and exploratory behavior (11) and in order to obtain data more directly related to exploratory behavior, we also tested the rats in an open-field, a more suitable model for the investigation of this kind of processes. A report of these data is the purpose of the present paper.

Adult male Wistar rats (194-230 g) from the animal house of the University of São Paulo at Ribeirão Preto were used. Rats had free access to food and water and were housed in groups of six to a cage on a 12:12-h darklight cycle (lights on at $7 \mathrm{am}$ ). Animals were divided randomly into three groups: 1) nonoperated controls $(\mathrm{N}=12), 2)$ saline-treated rats $(\mathrm{N}=12)$ that were injected with $0.5 \mu 1$ saline into the medial septum, and 3) SAPtreated rats $(\mathrm{N}=15)$, which were injected with SAP into the medial septum. Rats in the saline and SAP groups were anesthetized with sodium pentobarbital (Hypnol, Cristália, Itapira, SP, Brazil) and placed in a stereotaxic apparatus. The scalp was opened and a single hole was drilled over the medial septum coordinates (anteroposterior $=0.6$, dorsoventral $=6.5$ and mediolateral $=0.0$ ) (15). A single injection of either SAP (237.5 ng, Chemicon International Inc., Temecula, CA, USA) or $0.9 \%$ saline was delivered into the medial septal nucleus in a total volume of $0.5 \mu 1$ as described by Walsh et al. (14). Solutions were infused slowly through a 30 gauge dental needle attached to a Hamilton $5-\mu 1$ syringe. The syringe was mounted on a Harvard Apparatus microinjection unit and the rate of infusion delivery was $0.25 \mu \mathrm{l}$ per $\mathrm{min}$. The dental needle was left in place for an additional $2 \mathrm{~min}$ following each injection to allow for adequate diffusion. Animals were allowed 12 days to recover from surgery before the behavioral testing.

A $120-\mathrm{cm}$ square open-field with an opaque dark brown Formica floor surrounded by $50-\mathrm{cm}$ high wooden walls was used for the behavioral test. The tests were carried 
out in a room lit by a $60-\mathrm{W}$ light bulb $1.75 \mathrm{~m}$ above the center of the open-field. The experimental sessions were recorded by a video camera interfaced with a monitor and a videocassette recorder in an adjacent room. In order to record locomotor activity on line, the image of the open-field on the monitor screen was divided into thirty-six $20-\mathrm{cm}$ squares. Each rat was placed in the central area and allowed to freely explore for $5 \mathrm{~min}$ and every time both hind paws entered one square, a crossing was recorded. Total distance run by the animals was estimated from the number of squares crossed. After each test, the open-field was cleaned with a solution of $20 \%$ ethanol and then dried with a cloth. The total number of square crossings and the time spent in the central and peripheral areas (all squares next to the walls) were recorded on line. Rearing and grooming were also analyzed in terms of the place of occurrence, i.e., center or periphery. All measures were also analyzed minute by minute.

At the completion of behavioral testing rats were sacrificed by decapitation and regional acetylcholinesterase (AChE) activity was assessed according to the method of Ellman as modified by Augustinsson et al. (16). Briefly, hippocampus, frontal cortex and septum were dissected out and homogenized in $1 \%(\mathrm{w} / \mathrm{v})$ 0.1 M PBS, $\mathrm{pH}$ 7.3. The homogenate was then centrifuged for $15 \mathrm{~min}$ at $10,000 \mathrm{~g}$ and AChE activity was determined in the resulting supernatant. Aliquots of $150 \mu \mathrm{l}$ of the supernatant were incubated in $0.1 \mathrm{M}$ PBS containing $0.2 \mathrm{mM} \mathrm{4-4-}$ dithiodipyridine for $3 \mathrm{~min}$ at $37^{\circ} \mathrm{C}$. Then acetylthiocholine $(1 \mathrm{mM})$ was added to a final volume of $1 \mathrm{ml}$. Enzymatic activity was assessed by determining absorbance at 324 nm using a Varian DMS 80 spectrophotometer (Norwalk, CT, USA). Absorbance was measured in triplicate in each tissue sample 1,2 , and 3 min after the addition of acetylthiocholine. Statistical analysis was used to determine the differences in mean AChE activity during the third minute.

Since there were no differences between non-operated and saline-injected rats, these groups were combined into a single one, as of now designated as control. Statistical analysis using $t$-tests for independent samples showed that injection of SAP into the medial septum significantly decreased AChE activity in the hippocampus $\left(\mathrm{t}_{[35]}=21.7, \mathrm{P}<0.001\right)$, frontal cortex $\left(\mathrm{t}_{[32]}=8.2, \mathrm{P}<0.001\right)$ and septum $\left(\mathrm{t}_{[33]}=6.7, \mathrm{P}<0.001\right)$.

Behavioral comparisons of group means using the Mann-Whitney rank sum test to compare different groups (control x SAP) showed that intraseptal injection of SAP induced a significant decrease in the total distance run in the open-field (Figure 1). Further analyses showed that this general reduction was due to reductions in the distance run in both central $(\mathrm{T}=196.5, \mathrm{P}=0.003)$ and peripheral areas $(\mathrm{T}=225.5, \mathrm{P}=0.03)$ (Figure 1). On the other hand, SAP-treated rats

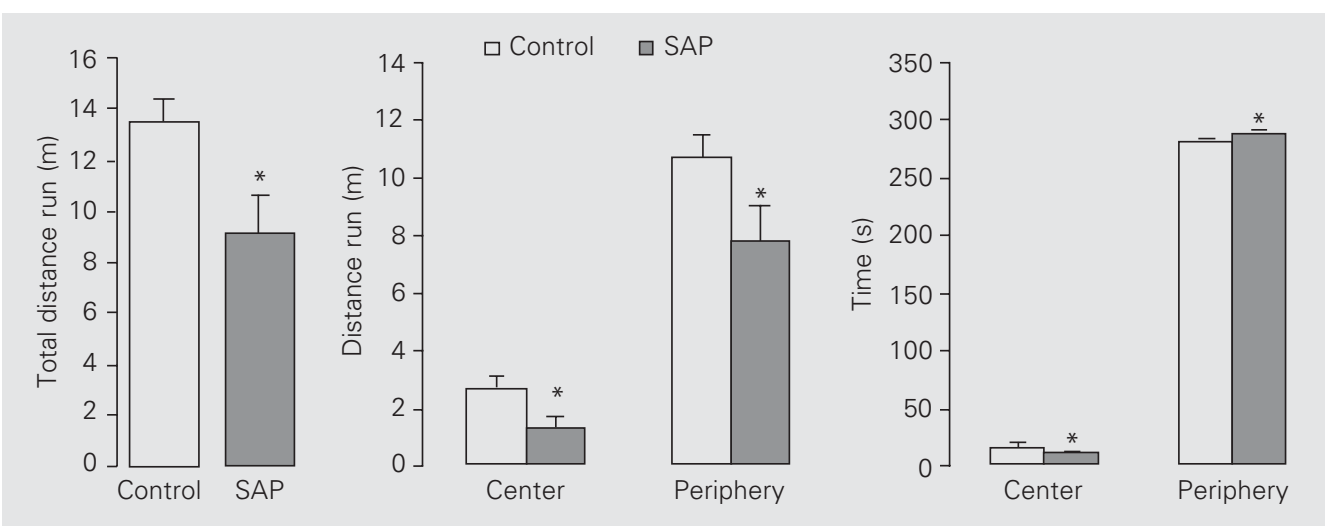

Figure 1. Effect of intraseptal lesion of the cholinergic component of the rat septohippocampal system. The figure reports total distance and distance run in the center and periphery (mean \pm SEM) of an open-field by control and 192 lgG-saporin (SAP)-treated rats during a 5-min session. ${ }^{*} \mathrm{P}<0.05$ compared to control (Mann-Whitney rank sum test). 
spent significantly more time in the peripheral area $(\mathrm{T}=371.5, \mathrm{P}=0.04)$ and less time in the central area $(\mathrm{T}=223.0, \mathrm{P}=0.03)$ when compared with the control animals (Figure 1). Finally, rearing and grooming did not differ between SAP-treated and control groups.

Additionally, analysis by the Wilcoxon signed rank test to compare different areas of the open-field (center $\mathrm{x}$ periphery) showed that control and SAP animals exhibited the standard profile of anxiety-related behaviors when placed in the open-field for the 5-min test session (10). They avoided entry into the central area $(\mathrm{W}=300, \mathrm{P}<0.001)$ and spent little time there $(\mathrm{W}=300, \mathrm{P}<0.001)$ (Figure 1).

Finally, to compare behavioral effects along the session in a minute-by-minute fashion we used the Friedman rank sum test for repeated measures followed by all pairwise multiple comparison procedures (StudentNewman-Keuls method). The analysis revealed that the control group showed a significant and gradual decrease in total $\left(\chi^{2}=\right.$

Figure 2. Effect of intraseptal lesion of the cholinergic component of the rat septohippocampal system. The figure reports total distance and distance run in the center and periphery (mean \pm SEM) of an open-field by control and 192 lgG-saporin (SAP)-treated rats during each minute of a 5 -min session. o $<<0.05$ compared to the respective first minute (Dunnett's test); ${ }^{*} P<0.05$ compared to control (Mann-Whitney rank sum test).

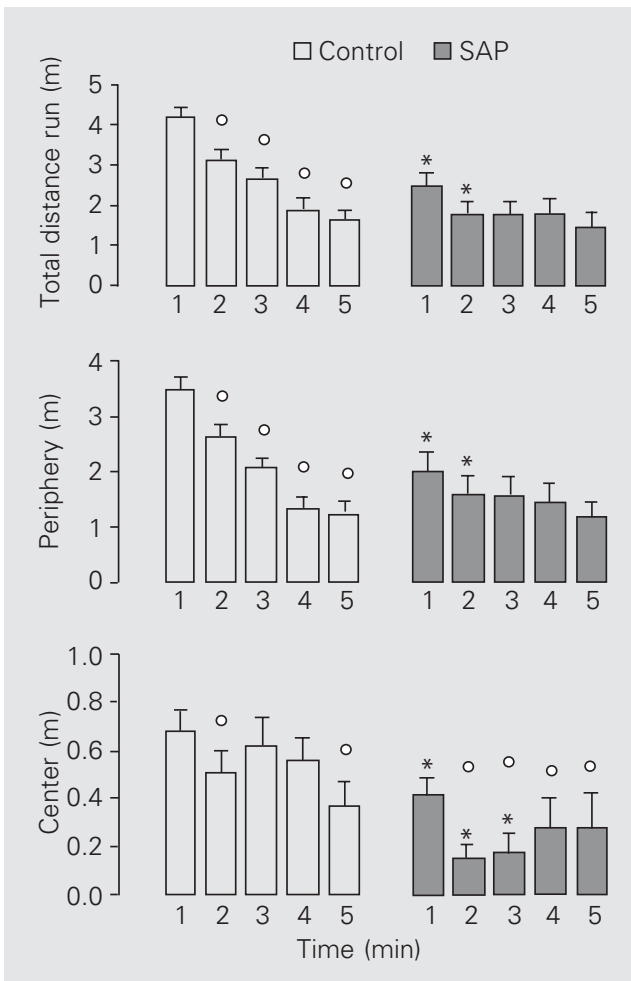

46.8, $\mathrm{P}<0.001)$, peripheral $\left(\chi^{2}=60.9\right.$, $\mathrm{P}<0.001)$ and central $\left(\chi^{2}=12.6, \mathrm{P}=0.013\right)$ distance run along the session; SAP-treated animals, on the other hand, did not exhibit such a gradual activity reduction along the session but instead tended to explore uniformly, except in the central area $\left(\chi^{2}=19.3\right.$, $\mathrm{P}<0.001$ ) (Figure 2).

SAP-treated animals exhibited a shorter distance run (total: $\mathrm{t}=181.0, \mathrm{P}<0.001$; periphery: $\mathrm{t}=184.5, \mathrm{P}<0.001$; center: $\mathrm{t}=223.5$, $\mathrm{P}=0.028)$ compared to control rats in the first minute (Figure 2). In the second minute, SAP-treated animals exhibited a shorter distance run (total: $\mathrm{t}=200.0, \mathrm{P}=0.004$; periphery: $\mathrm{t}=210.5, \mathrm{P}=0.010$; center: $\mathrm{t}=235.5$, $\mathrm{P}=0.064)$. Finally, in the third minute SAPtreated animals explored the central area less than the control group $(\mathrm{t}=202.0, \mathrm{P}=0.005)$.

In general, the SAP-treated group exhibited less activity at the beginning of exposure to the open-field and less habituation of exploratory behavior over the 5-min test session than the control group.

Intraseptal injection of SAP produced specific behavioral effects associated with an extensive decrease in AChE activity in the hippocampus and, to a lesser extent, in the frontal cortex. AChE activity in the hippocampus has been shown to correspond to the innervation of hippocampal neurons by terminals originating in the cell bodies in the medial septum (17). Moreover, histochemical studies have demonstrated that disrupting septohippocampal connections by septal lesions (18) or fimbria-fornix transections decreases $\mathrm{AChE}$ in the hippocampus (17).

Previous reports have shown that the dose of SAP used in the present study $(237.5 \mathrm{ng} /$ $0.5 \mu \mathrm{l})$ has no long-term effect on the levels of serotonin, norepinephrine, dopamine, or their metabolites in the hippocampus, frontal cortex, or striatum (14). In addition, this dose of SAP has been shown to produce a delay-dependent deficit in a spatial working memory task (14) without affecting attentional processes in a latent inhibition 
paradigm (19).

The data presented here demonstrate that intraseptal SAP does not affect the distance run in the central area of the open-field, considered by some a measure of anxiety $(8,9)$. In fact, SAP-treated rats exhibited a clear reduction in exploratory behavior in all areas of the open-field. As mentioned above, electrolytic lesions of the medial septum abolish the motivation to initiate exploration of an open-field voluntarily accessible from the home cage (5) and decrease the exploration of novel objects in an open-field (6). The data of the present experiment allow us to attribute the deficits in exploratory behavior to the selective destruction of the cholinergic component of the septohippocampal pathway and not to other neurotransmitter systems also present in this pathway.

Additionally, the decrease in exploratory behaviors is consistent with previous reports of the role of cholinergic processes in the modulation of locomotor activity and in the exploratory repertoire of rodents. For example, infusion of the muscarinic agonist carbachol into the septal area or into the hippocampus increases the frequency of behaviors associated with exploration, such as rearing, ambulation, scanning, and movements of the vibrissae $(20,21)$. These behavioral effects were reversed by a small dose of atropine, suggesting that muscarinic receptors in the hippocampus play a crucial role in the initiation of locomotor activity. Literature data about SAP lesions plus the results obtained in the present study suggest that the septohippocampal cholinergic system, together with other cholinergic projections probably originating in the tegmental pedunculopontine and laterodorsal nuclei (see Ref. 22 ), contribute to the modulation of behaviors associated with exploration.

On the other hand, the persistence of exploration by SAP-treated animals (but not by controls) throughout the 5 -min period of testing allows us to infer a deficit in spatial memory processes, as reported by us in the elevated plus-maze (11). Intraseptal injection of SAP $(2,14)$ has been shown to produce deficits in other spatial memory tasks, indicating alterations in goal-directed exploratory behavior. The results of the present study suggest that septohippocampal cholinergic mechanisms contribute to at least two critical behavioral processes, one related to the motivation to explore new environments and the other related to the acquisition and storage of spatial information (i.e., spatial memory).

\section{Acknowledgments}

The authors are indebted to Juan Carlos Martinez, Universidad de la Sabana, Colombia, for help with the behavioral experiments.

\section{References}

1. Dutar MH, Bassant MH, Senut MC \& Lamour $Y$ (1995). The septohippocampal pathway: structure and function of a central cholinergic system. Physiological Reviews, 75: 393-427.

2. Shen J, Barnes CA, Wenk GL \& McNaughton BL (1996). Differential effects of selective immunotoxic lesions of medial septal cholinergic cells on spatial working and reference memory. Behavioral Neuroscience, 110: 1181-1186.

3. McNaughton N \& Gray JA (2000). Anxiolytic action on the behavioural inhibition system implies multiple types of arousal contribute to anxiety. Journal of Affective Disorders, 61: 161-176.

4. Walsh TJ \& Chrobak JJ (1991). Animal models of Alzheimer's disease. Role of hippocampal cholinergic system in working memory. In: Dachowsky L \& Flaherty C (Editors), Current Topics in Animal
Learning, Brain, Emotion and Cognition. Lawrence Erlbaum, Hillsdale, NJ, USA, 347-379.

5. Kohler C \& Srebro B (1980). Effects of lateral and medial septal lesions on exploratory behavior in the albino rat. Brain Research, 182: $423-440$.

6. Myhrer T (1989). Exploratory behavior and reaction to novelty in rats: effects of medial and lateral septal lesions. Behavioral Neuroscience, 103: 1226-1233.

7. Lee EHY, Lin YP \& Yin TH (1988). Effects of lateral and medial septal lesions on various activity and reactivity measures in rats. Physiology and Behavior, 42: 97-102.

8. Roth KA \& Katz RJ (1979). Stress, behavioral arousal, and open-field activity - a reexamination of emotionality in the rat. Neuroscience 
and Biobehavioral Reviews, 3: 247-263.

9. Lee EHY, Tsai MJ \& Chai CY (1986). Stress selectively influences central region activity of mice in an open-field. Physiology and Behavior, 37: 659-662.

10. Lee EHY, Tang YP \& Chai CY (1987). Stress and corticotrophin releasing factor potentiate center region activity of mice in an openfield. Psychopharmacology, 93: 320-323.

11. Lamprea MR, Cardenas FP, Silveira R, Morato S \& Walsh TJ (2000). Dissociation of memory and anxiety in a repeated elevated plus maze paradigm: forebrain cholinergic mechanisms. Behavioural Brain Research, 117: 97-105.

12. Wiley RG \& Lappi DA (1994). Suicide Transport and Immunolesioning. RG Landes Co., Austin, TX, USA.

13. Wiley RG \& Lappi DA (1992). Neural lesioning with ribosome-inactivating proteins: Suicide transport and immunolesioning. Trends in Neurosciences, 15: 285-290.

14. Walsh TJ, Herzog CD, Gandhi C, Stackman RW \& Wiley RG (1996). Injection of IgG 192-saporin into the medial septum produces cholinergic hypofunction and dose-dependent working memory deficits. Brain Research, 726: 69-79.

15. Paxinos G \& Watson C (1997). The Rat Brain in Stereotaxic Coordinates. 3rd edn. Academic Press, San Diego, CA, USA.

16. Augustinsson KB, Eriksson $H$ \& Faigersson $Y$ (1978). A new ap- proach to determining cholinesterase activities in samples of whole blood. Clinica Chimica Acta, 89: 239-252.

17. Lewis PR, Shute CCD \& Silver A (1967). Confirmation from choline acetylase analysis of a massive cholinergic innervation to rat hippocampus. Journal of Physiology, 191: 215-224.

18. Mellgren SI \& Srebro B (1973). Changes in acetylcholinesterase and distribution of degenerating fibers in the hippocampal region after septal lesions in the rat. Brain Research, 52: 19-36.

19. Dougherty KD, Salat D \& Walsh TJ (1996). Intraseptal injections of the cholinergic immunotoxin 192-lgG saporin fails to disrupt latent inhibition in a conditioned test aversion paradigm. Brain Research, 736: 260-269.

20. Flicker C \& Geyer MA (1982). Behavior during hippocampal microinfusions II: muscarinic locomotor activation. Brain Research, 257: 105-127.

21. Monmaur P, Sharif A \& M'harzi M (1997). Involvement of septal muscarinic receptors in cholinergically mediated changes in rat rearing activity. Pharmacology, Biochemistry and Behavior, 58: 577-582.

22. Brudzynski SM, Wu M \& Mogenson GJ (1988). Modulation of locomotor activity induced by injection of carbachol into the tegmental pedunculopontine nucleus and adjacent areas in the rat. Brain Research, 451: 119-125. 